MADGU. Mundo, Arquitectura, Diseño gráfico y Urbanismo. NÚM 07, 2021 ISSN: 2594-1208, PP. 44-59

Recibido: 05 de Noviembre de 2020

Aceptado: 20 de Diciembre de 2020

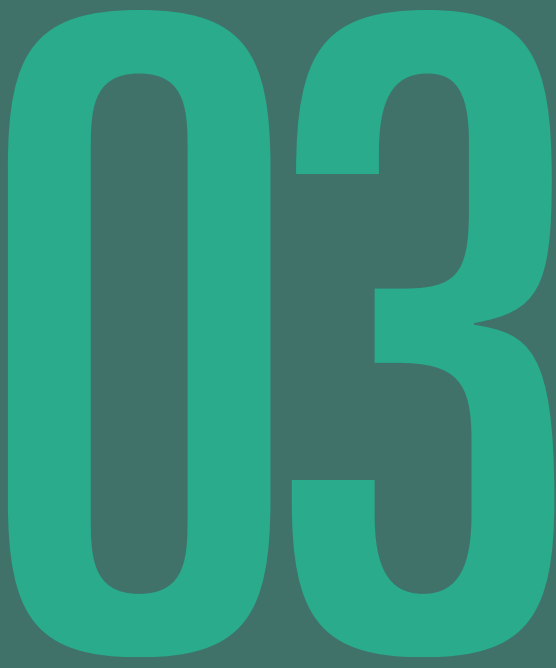

\title{
DINÁMICAS URBANAS, CONSTRUCCIÓN Y EJERCICIO DE CIUDADANÍA EN EL ESPACIO PÚBLICO
}

\section{Urban dynamics, construction and exercise of citizenship in public space}

\author{
Venancia Tristan Bernal \\ https://orcid.org/0000-0002-1087-0734 \\ bernal76_9@hotmail.com
}

Profesora de la Facultad de Arquitectura de la Universidad Autónoma de Nuevo León, UANL. Estudiante de la Maestría en Ciencias con

Orientación en Asuntos Urbanos por la misma institución.

Jesús Manuel Fitch Osuna

https://orcid.org/0000-0003-2795-3357

jesus.fitchos@uanl.edu.mx

Profesor e Investigador Titular, Estudios urbanos e inmobiliarios, Facultad de Arquitectura, UANL. Miembro del Sistema Nacional de

Investigadores 
Palabras clave: Espacio público, ciudad, ciudadanía, dinámicas urbanas Keyword: Public space, city, citizenship, urban dynamics

\section{Resumen:}

El estudio sobre la experiencia de la vida urbana cotidiana permite hacer reflexiones y cuestionamientos sobre diversos fenómenos, entre ellos los derivados de lo urbano. La ciudadanía y el espacio público contemporáneo son los temas que se trabajaron en el presente documento con el fin de comprender algunos aspectos ligados al reconocimiento del derecho de uso, y no como un conjunto de prácticas sociales, culturales, politicas y económicas. Para ello se estableció el objetivo, que consideró el analizar los actores y procesos urbanos que intervienen en la construcción y ejercicio de la ciudadanía en el espacio público. A través de una revisión bibliográfica, se construyó un cuerpo teórico que permitió posteriormente ser trasladado a un caso concreto. Se consideró para ello el caso de colonia Mitras centro en la ciudad de Monterrey, México. El método que se empleó es cualitativo, procedente de la etnografía. Cabe destacar las técnicas de recopilación de información apoyadas en las teorías y métodos desarrollados por el Gehl Institute. Entre los resultados y conclusiones se consiguió generar las representaciones sociales de los habitantes a través de sus acciones, expresadas en las prácticas del espacio público en relación con la ciudadanía.
Abstract:

The study on the experience of everyday urban life allows for reflections and questions about various phenomena, including those derived from the urban. Citizenship and contemporary public space is the topic that was worked on in this document, in order to understand some aspects related to the recognition of the right of use, and not as a set of social, cultural, political and economic practices. For this, the objective was established, which considered analyzing the urban actors and processes that intervene in the construction and exercise of citizenship in public space. It is through a bibliographic review that a theoretical body was built, which later allowed it to be transferred to a specific case. The case of colonia Mitras centro in the city of Monterrey, Mexico was considered for this. The method that was used is qualitative, coming from ethnography. It is worth highlighting the information gathering techniques, supported by the theories and methods developed by the Gehl Institute. Among the results and conclusions, it was possible to generate the social representations of the inhabitants through their actions, expressed in the practices of the public space in relation to citizenship. 
Venancia Tristan Bernal

Jesús Manuel Fitch Osuna

\section{INTRODUCCIÓN}

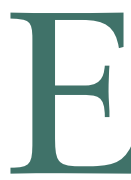

n México, tres de cada cuatro personas habitan en ciudades según el Censo de la CONAPO y SEDESOL presentado en el 2013. En cuanto a esto, se debe tener en cuenta que, según la ONU-Hábitat, en las próximas décadas gran parte del crecimiento demográfico en el país será urbano, lo cual indica que el país pasará de contar con 384 ciudades a contar con 961 en 2030. En esta proyección a futuro, las ciudades concentrarán el $83.2 \%$ de la población nacional y es muy probable que se trate de una población pobre, según la Organización.

Otro aspecto del crecimiento poblacional urbano es la demanda dentro de las ciudades y la necesidad de más empleo, más servicios y más vivienda. Esto genera problemáticas sociales, ambientales y económicas; además de un impacto en la calidad de vida diaria de los habitantes, el cual afecta negativamente su potencial de desarrollo económico y social.

La expansión urbana de la ciudad ha implicado una división social del espacio, la forma de socializar y un deterioro urbano, donde el espacio público es despreciado por el nuevo urbanismo y por el propio habitante. Refieren 
Ramírez y Safa (2010) que este fenómeno es distintivo de cualquier urbe con un modelo de ciudad segregativo que busca habilitar, construir o consolidar espacios urbanos adecuados para el comercio, las finanzas y la vivienda de las clases alta y media alta.

En la misma vertiente teórica, destacan García (1998), Borja (1997), Castells y Borja (2000) que las ciudades mexicanas se describen como conglomerados que sufren de un triple proceso negativo: disolución, fragmentación y privatización. Lo anterior contribuye a ampliar las distancias sociales y, en consecuencia, las ciudades se expanden con déficit de espacio público. Esto provoca que surjan espacios seudopúblicos y privatizados que sustituyen las funciones concernientes a los espacios públicos tradicionales.

Por consiguiente, surge el interés de estudiar las dinámicas urbanas, construcción y ejercicio de ciudadanía. El trabajo expuesto es parte sustancial de la investigación de Tristan (2020), titulada: Análisis del uso del espacio público desde la habitabilidad y dinámicas urbanas contemporáneas: el caso de colonia Mitras centro en la ciudad de Monterrey, en donde el objetivo es revisar teóricamente los conceptos que se asocian a las dinámicas, procesos urbanos y los actores que intervienen en la construcción y ejercicio de ciudadanía dentro del espacio público. Para esto, se parte del supuesto de que el espacio público se constituye como escenario para la construcción de la ciudadanía — cimentada socialmente por las formas de uso y apropiación del espacio- y se centra en el contexto de la ciudad contemporánea.

La primera parte del documento corresponde a una revisión teórica, organizada a partir de las siguientes cuestiones: ¿qué es la ciudadanía? y ¿cómo se construye en el espacio público? Estas interrogantes germinan de la variedad de interpretaciones de finales del siglo XX hasta la actualidad sobre lo que es ciudad, ciudadanía y espacio público, conceptos que han sido objeto de análisis en conjunto y por separado en distintos campos teóricos de las ciencias sociales y del urbanismo. 
La segunda parte describe la metodología, sustentada en las teorías desarrolladas por el Gehl Institute. Estas analizan las representaciones sociales de los habitantes a través de sus acciones, expresadas en las prácticas del espacio público en relación con la ciudadanía. Lo anterior se realiza mediante una observación meticulosa pero objetiva de los sucesos que acontecen, donde el investigador se convierte en un actor en la construcción de la descripción y del ambiente que observa y analiza. En síntesis, el estudio aborda — como punto central - el espacio público bajo la definición de ciudadanía, entendiéndolo como elemento generador de relaciones y prácticas sociales, además de espacio productor y de ejercicio de ciudadanía.

Dentro de la constante transformación del espacio público como lugar de consumo, se ha destacado que los cambios en la manera de consumir han alterado las posibilidades y las formas de ser ciudadano (Ramirez, 2009). Es por ello por lo que la calidad del espacio público se podrá evaluar por la intensidad e importancia de las relaciones que facilita. En este sentido, se debe dejar de considerar al espacio público como un indicador de condición urbana, y optar por que sea un indicador de calidad de ciudad.

\section{LA CONCEPTUALIZACIÓN DE CIUDADANÍA DESDE SU PRINCIPIO, EL CIUDADANO}

El concepto de ciudadanía está relacionado con un principio básico: el ciudadano, término que procede del vocablo latino cives (ciudadano) y que designa la posición del individuo en la civitas (ciudad). No obstante, el concepto conlleva una condición tanto jurídica como política y presenta cierta dualidad: por un lado, inclusivo y, por el otro, excluyente, al considerar solo a aquellos que tienen derecho por nacionalidad, sin atender a determinados grupos de habitantes donde el derecho se distingue entre originarios, habitantes y vecinos ${ }^{1}$.

1 En el Distrito Federal, el derecho distingue entre originarios, habitantes, vecinos; siendo originarios los que nacen en el territorio del Distrito Federal, habitantes quienes residen ahi, y vecinos, aquellos que viven en ese territorio por más de seis meses.; concerniente a las disposiciones del Estatuto de Gobierno del Distrito Federal, publicado en el Diario Oficial de la Federación del 26 de julio de 1994; así como a lo previsto en la Ley de Participación Ciudadana del Distrito Federal, publicada en la Gaceta Oficial del Distrito Federal el 27 de mayo de 2010. 
Para autores como García (1995), Castel (1997) y Alonso (1999), la exclusión refleja la perdida de condición de ciudadanía de una parte de la población. El nacimiento de la ciudadanía se produce en la Antigua Grecia con la relación entre ciudad y Estado, donde el ciudadano forma parte del Gobierno de la ciudad, la cual es reservada a una pequeña fracción de habitantes. Algo semejante ocurre en la Antigua Roma, donde la expresión ciudadanía se vinculó a la relación de un individuo con su ciudad (de la civitas) y donde la ciudadanía era un privilegio de los hombres libres, aquellos que podían y debían contribuir económica o militarmente al sostenimiento de la ciudad (Arango, 2006).

En La política, el pensador griego Aristóteles (trad. en 1941) define al hombre como un ser naturalmente sociable, un animal social o político, mas no concibe al ser humano fuera de la ciudad. Para él, el hombre es un ser social, un individuo que necesariamente debe vivir en un ámbito comunitario. En esta línea, Marshall y Bottomore (1992) señalan que el ciudadano se constituye como aquel que tiene derecho a tener derechos en un status de tres dimensiones: la civil, la política y la social. Por el contrario, Arendt (2005) lo considera a partir de su capacidad de acción en el espacio público, es decir, no será suficiente cumplir con los parámetros legales ${ }^{2}$ que permiten a un individuo ser ciudadano y donde ser ciudadano se convierte en una actitud deseada.

De manera que, contextualizado a la actualidad el concepto de ciudadano, este ha evolucionado a través de varias vertientes teóricas, lo cual se puede notar con lo que plantean Marshall y Bottomore (1992) quien realizan una crítica a la teoría y a la práctica de la ciudadanía tradicional. En ella, la ciudadanía se presenta con una dualidad, la igualdad legal y la política; y, por otro lado, con una desigualdad material. Esto deriva en la propuesta de no limitar al concepto sólo a los derechos políticos y propone, además, integrar la dimensión social en acuerdo con Turner (1990), quien establece una clasificación binominal del concepto.

2 Se reserva el estatus de ciudadano a los varones y mujeres que: tengan la calidad de mexicanos, hayan cumplido 18 años, y posean la calidad de vecinos $u$ originarios. 
La llamada ciudadanía pasiva, conocida también como ciudadanía desde arriba, representa la responsabilidad del Estado y a una ciudadanía conservadora. La ciudadanía activa, llamada también ciudadanía desde abajo, se representa a partir de la acción y ejercicio de habitar el espacio público como lugar de convivencia, donde se desarrollan prácticas sociales, culturales, políticas y económicas en el espacio. Acorde con la multidimensionalidad del espacio público: la dimensión jurídico-política, la social, funcional y la cultural, resulta necesario admitir que distintos autores suponen estas dimensiones como válidas; pero contemplan más dimensiones complementarias e interdependientes como la dimensión física, la dimensión de seguridad, la económica y la ambiental.

\section{ESPACIO PÚBLICO-CIUDAD Y CIUDADANÍA}

Hablar de espacio público y ciudadanía sin antes reflexionar en la coexistencia de ambos sería un error. La relación espacio público-ciudadanía no es terminología simplemente; la ciudad ofrece condiciones para construir y ejercer la ciudadanía, la hace tangible. Es donde los habitantes se transforman a ciudadanos, accediendo a los bienes y servicios, conquistando sus derechos al hacerlos efectivos y aquí radica o se determina la calidad de la ciudadanía.

Para Borja y Muxi (2000) la ciudad es, ante todo, un espacio público y entienden a la ciudadanía como igualdad de derechos sociales, políticos, económicos y culturales espacializados en el espacio público, donde la ciudadanía se ejerce en el uso y la apropiación, denotando una pertenencia a la comunidad, con iguales derechos y responsabilidades. De ello resulta necesario admitir que la ciudad es fundamental para el desarrollo de la ciudadanía; la manera de vivir, la acción en el espacio público, se convierte en punto clave para entender la construcción y ejercicio de ciudadanía.

Lo anterior no quiere decir que el reunirse en el espacio público es condición suficiente para la construcción de ciudadanía. Es necesario además que los habitantes interactúen, desde la posición que argumenta Habermas (1986) que la acción ciudadana, si bien necesita un escenario, nunca sería posible sin el ejercicio del logos (la palabra), la acción comunicativa. 


\section{PROCESO METODOLÓGICO}

El diseño del método se definió a partir de las condicionantes de la investigación cualitativa, específicamente la etnografía, la cual tiene como objetivo hacer una descripción minuciosa de los sucesos que acontecen, para con ello retratar las visiones de sus integrantes de manera individual y colectiva (Narváez y Carmona, 2017). Mediante una observación objetiva, el investigador se convierte en un actor en la construcción de la descripción y del ambiente que observa y analiza, donde la validez de los estudios cualitativos se encuentra en lo particular, en lo cambiante, en lo versátil, dependiente de la situación del hecho tal cual es y de lo cotidiano (Narváez y Carmona, 2017). Es por ello que los datos se recolectan, con la finalidad de analizarlos y comprenderlos para responder las preguntas de investigación y generar conocimiento.

La descripción permitió analizar las dinámicas, procesos urbanos y los actores que intervienen en la construcción y ejercicio de ciudadanía dentro del espacio público. A esto se sumó un trabajo previo de diagnóstico de cada uno de los objetos de estudio, cotejado permanentemente con información bibliográfica, datos estadísticos de la zona, fuentes cartográficas, características demográficas y socioeconómicas, para elaborar un perfil sobre la población y territorio, el cual permitió valorar en conjunto los objetos de estudio.

Conviene subrayar que el estudio manejó un método de muestreo no probabilístico por conveniencia, el cual es apoyado con la metodología teórica-grafica desarrollada por el Gehl Institute como herramienta fundamental de trabajo y consta de técnicas de compilación de información. Esto permitió una aproximación a la realidad social en el fenómeno que se estudió, posibilitando una visión total de la realidad y tomando en cuenta el contexto sin fragmentar (Gehl, 2006). A través de los sentidos se realizó una observación de los hechos y realidades sociales presentes, a través de observación al sujeto de estudio en un contexto real, en donde desarrolla sus actividades, lo que posibilita el acercamiento interpretativo. 
De acuerdo con Gehl (2006) las actividades de la vida cotidiana se dividen en tres. La primera consiste en las actividades diarias o necesarias, la cuales corresponden a trabajar, ir a la escuela y comprar. La segunda tiene que ver con lo recreativo u opcionales, tal como salir a caminar, leer en una banca, reunirse con personas. Y en tercer lugar están las actividades sociales, como convivir, participar en alguna tarea colectiva o conversar con extraños.

Posterior al planteamiento se prosiguió a la recopilación de información, para lo cual se siguió un proceso integrado por tres instrumentos bien definidos y correlacionados con las variables e indicadores estudiados:

1. La observación sistemática no intrusiva.

2. La caracterización del espacio público y de los sujetos de estudio.

3. Encuestas aplicadas en sitio.

\section{DESCRIPCIÓN DE INSTRUMENTOS Y TÉCNICAS}

Los instrumentos y técnicas definidos para el estudio son acordes al planteamiento y se han validado teóricamente a partir de los argumentos previos. La primera técnica, la observación sistemática no intrusiva y la caracterización del sitio y del sujeto, consiste en llevar el conteo y mapeo de los siguientes puntos:

- Conteo de personas en movimiento: ayudó a entender cuántas personas se mueven por el espacio, cómo y cuándo.

- Conteo de edad y género: permitió visualizar quién utiliza el espacio.

- Mapeo de actividades estáticas: proporcionó una imagen de las actividades que las personas eligen realizar en el espacio.

- Inventario de un lugar: permitió la caracterización del lugar.

- Documentar el proceso con fotografías y mapeo.

La observación sistemática no intrusiva y la caracterización del sitio y del sujeto de estudio se llevaron a cabo en un periodo que comprende del mes de enero a abril del año 2019, donde la observación no intrusiva fue básica en la recolección de información. Se inició con conteos de edad y género, de peatones en movimiento y mapeo de actividades estáticas en la zona, a la par 
del inventario del lugar, lo cual permitió contar con información detallada sobre los lugares en los que la gente camina y las actividades que llevan a cabo cuando están estáticos.

Este instrumento constó de un análisis secuencial en cada uno de los objetos de estudio a diferentes horas del día (mañana, tarde, noche) y distintos días de la semana —incluyendo días festivos-. Se mapeó dicha información y se registró en una ficha que ayudó a la posterior clasificación de la información según la dimensión, ya sea la física o la social.

El tercer instrumento de la metodología propuesta corresponde a las encuestas $^{3}$, para ello se contó con el apoyo de estudiantes de la Facultad de Arquitectura (UANL) del séptimo semestre de la carrera de Arquitectura. Previo a la aplicación del instrumento, se les capacitó en la metodología y se les facilitó el plano de ubicación, así como los formatos de mapeo de los cuatro objetos de estudio. Se les informó del contexto inmediato de la zona, la identificación dentro del estudio, además de realizar una prueba piloto.

Las encuestas se llevaron a cabo en el periodo del $1^{\circ}$ al 31 de mayo del año 2019; se aplicaron, en total, a 275 usuarios. Una parte fueron residentes (viven en la colonia y hacen uso del espacio) y otros, no residentes (ocupan el espacio solo para tránsito o se encontraban en el sitio el día de la aplicación del instrumento).

\section{DELIMITACIÓN ESPACIAL DE LA ZONA DE ESTUDIO}

La delimitación espacial es esencial en el abordaje de los fenómenos urbanos, ya que permite propiciar los alcances y limitaciones geográficos del estudio. Para el presente trabajo se estableció la escala de barrio como ámbito de estudio. Este corresponde a la colonia Mitras centro en la ciudad de Monterrey, en donde se ubican los cuatro espacios públicos que forman parte del análisis; son parques y plazas que se localizan en el corazón de la colonia (ver figura 1).

3 se dividen en dos secciones, la primera para el no residente que consta de 10 items y la segunda para el residente con 38 items aplicados en el sitio y de forma individual donde el tiempo de aplicación fue variable de cinco minutos y 15 minutos respectivamente. 
Mitras centro comenzó su formación en la década de los cuarenta del siglo XX. Fue entonces que la ciudad de Monterrey experimentó una dinámica productiva, un crecimiento económico y poblacional derivado de la llegada de la industria pesada, la fundición, el vidrio, cemento y la cerveza. Esto modificó la forma de creación de riqueza y su distribución en la ciudad y atrajo movimientos migratorios del campo, así como de inversionistas y trabajadores de otras ciudades o de otros países, lo cual derivó en la creación de barrios obreros y zonas industriales.

Para la década de los cuarenta, la población se había duplicado con relación a inicios de siglo XX. Esto promovió la proliferación de zonas habitacionales residenciales y el incremento de las necesidades de la creciente sociedad regiomontana, lo que inició la expansión territorial. La ciudad perdía ya su imagen provinciana, se diversificaba y un perfil urbano acabaría por formarse. La colonia se convertía con el tiempo en el prototipo y sueño residencial de profesionistas con familia, con un mediano poder adquisitivo.

La colonia destacó por su extensión, su estilo y por ser donde se consolidaba la clase media regiomontana, reflejo de prosperidad y expansión de la ciudad en esa época. Las Mitras — como se publicitó en aquellos años- ofrecía un atractivo por el trazo de sus calles; todos los servicios los ofrecía en un fraccionamiento moderno, conformado por una calzada central que atravesaba la colonia en toda su longitud, y que le permitía ser punto neurálgico de la ciudad hasta ese momento, debido a que su calzada conectaba hacia colonias como El Obispado y con el centro de la ciudad.

La colonia está situada en parte del territorio municipal que se divide en cinco delegaciones, a su vez subdivididas en distritos ${ }^{4}$.

Esto corresponde a la delegación centro y el distrito Mitras. La zona se caracteriza a partir de la década de los noventa por ser un centro expulsor de habitantes, a nuestros días la tendencia continúa.

4 El municipio de Monterrey está dividido en cinco unidades territoriales que se denominan delegaciones y que a su vez se subdividen en distritos; Delegación centro, norte, poniente, sur y Huajuco. Plan de Desarrollo Urbano del Municipio de Monterrey 2013-2025. 
El lugar ha sido escenario de fenómenos y notables transformaciones que repercuten en el uso y apropiación del espacio. Uno de los primeros fenómenos que impactó en la colonia fue la construcción del sistema Metro, a inicios de la década de los noventa, en la totalidad de su calzada principal y que partió en dos la zona. Cabe mencionar que son estaciones de tipo elevado, acompañados de metrobus y transmetro como sistemas de conexión entre ellos.

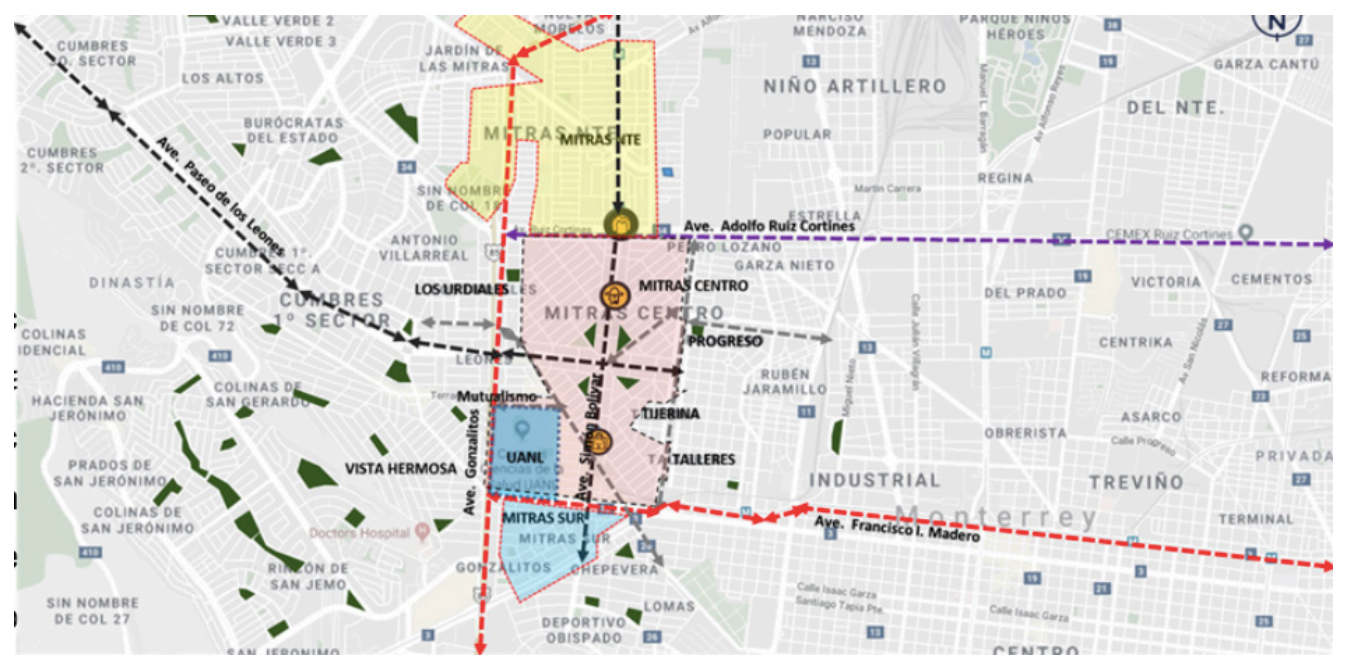

Figura 1: Contexto del caso de estudio, Fuente: Elaboración propia

La línea 1 tiene una longitud total de 17 kilómetros con 19 estaciones, de los cuales tres kilómetros y tres estaciones (Mitras, Simón Bolívar y Hospital) transitan por la colonia y a la vez la dividen. A partir de la construcción del metro se registraron cambios en el uso de suelo, principalmente de habitacional a uso mixto. Las zonas habitacionales paulatinamente fueron desplazadas por usos comerciales, lo que resultó en altos grados de percepción de inseguridad, transporte colectivo de mala calidad y la proliferación de comercio formal e informal.

En años recientes, los problemas se extendieron y se derivó en segregación y gentrificación en la zona, déficit de espacio público y mala calidad de este. Así mismo, surgió la formación de una población flotante (no residentes), es decir, trabajadores con empleo permanente derivado de los cambios de uso de suelo anteriormente descritos y alumnos de diferentes instituciones como el 
Campus de ciencias en la salud $\mathrm{UANL}^{5}$ — que alberga a 22,575 usuarios entre estudiantes y trabajadores-, que no son residentes de la colonia y representan un impacto mayor que la propia población residente.

\section{CONCLUSIÓN}

En el proceso de revisión y análisis teórico se pueden destacar aspectos conceptuales, así como una necesidad de replantear o adecuar los conceptos. El espacio público contemporáneo es utilizado de variadas formas, su dimensión espacial permite o pretende con ello satisfacer necesidades relacionadas con la calidad de vida urbana. Sin embargo, en la actualidad su uso es crítico, es un reflejo de la propia evolución de la ciudad, la sociedad y su historia, además de la relación entre las condiciones de habitabilidad y el comportamiento urbano del habitante en la ciudad.

En las últimas décadas la demanda del espacio público ha mutado por parte del usuario-habitante de la ciudad contemporánea, quien busca un equilibrio físico, psicológico y social pero no en el espacio público. Esta demanda es influida directamente por la vida cotidiana o las propias dinámicas urbanas que conllevan cambios significativos en rutinas, patrones de conducta, asociados al incremento o proliferación de espacios privados y al aumento de asistencia por parte del ciudadano a estos, sumado a la diversidad de actividades que el espacio privado permite, pero sin despreciar la calidad y la intensidad de las relaciones que este facilita.

De ahí que el análisis realizado permite dirigir la atención a las actividades que se estructuran en el espacio y al mismo tiempo abordar las problemáticas en él. Es posible identificar que la relación usuario-espacio se manifiesta inicialmente en el sitio donde se habita, asociado al ámbito psicológico en relación con los sentidos, más aun, a la creación de una identidad tanto individual como grupal en el espacio. Por tales motivos, el uso del espacio público se encuentra entre los sectores que más influyen en el proceso social, donde las

5 Campus de Ciudad Universitaria, con una extensión de 291,855.00 m2, tiene en su sede cinco facultades relacionadas con el área de la salud: Odontología, Medicina, Enfermería, Psicología y Salud Pública y Nutrición, así como el Hospital Universitario "Dr. José Eleuterio González" y una biblioteca especializada del área de la salud. 
tendencias actuales introducen o impactan directamente en el uso o desuso del espacio y al mismo tiempo a la composición de las ciudades contemporáneas frente al proceso de urbanización.

De ahí que el método utilizado permite tener ventajas al visualizar las dinámicas, procesos urbanos y los actores que intervienen en la construcción y ejercicio de ciudadanía dentro del espacio público. Además, ofrece una visión más clara de la caracterización del espacio y del usuario, introduciéndonos en la vida cotidiana y con ello plasma enfoques de manera individual y colectiva de sus integrantes. Cabe mencionar que permite al investigador ser parte y al mismo tiempo describir el ambiente que observa y analiza. En síntesis, se puede ultimar que a partir del estudio se derivan elementos para definir pautas para el ajuste conceptual, así como la mejora metodológica en los procesos fenomenológicos del espacio público. 


\section{REFERENCIAS}

Alonso, L. (1999). Trabajo y ciudadanía, Estudios sobre la crisis de la sociedad salarial. Madrid: Trotta.

Arango, J. (2006). Ciudadanía, diversidad y civismo. Madrid: Centro de Estudios sobre Ciudadanía y Migraciones del Instituto Universitario Ortega y Gasset.

Arendt, H. (2005). La condición Humana. Barcelona: Paidós.

Aristóteles. (1941). La Política; Colección Austral. Buenos Aires: Espasa-Calpe.

Borja, J. (1997). Ciudadanía y espacio público. Barcelona: Electa.

Borja, J. y Muxi, Z. (2000). El espacio público, ciudad y cíudadania. Barcelona: Electa.

Castel, R. (1997). La metamorfosis de la cuestión social; Una crónica del salariado. Buenos Aires: Paidós.

Castells, M. y Borja, J. (2000). Local y global; La gestión de las ciudades en la era de la información. México: Taurus.

Consejo Nacional de población. (2013). La situación demografica de México. Recuperado de http://www.conapo.gob.mx/work/models/CONAPO/Resource/2468/2/ images/SDM_2013.pdf

García, C. (1995). Consumidores y ciudadanos; Conflictos multiculturales de la globalización. México: Grijalbo.

García, C. (1998). Las cuatro ciudades de México. En Néstor García Canclini (coord.), Cultura y comunicación en la ciudad de México. Primera parte. Modernidad y multiculturalidad: la Ciudad de México a fin de siglo. México: UAM/Grijalbo.

Gehl, J. (2006). La Humanización del Espacio Urbano. La vida social entre los edificios. Barcelona: Reverte. 
Narváez, A. y Carmona, G. (2017) Etnografía y Netnografía. Su aplicación para la Arquitectura y Urbanismo. México: Pearson.

Onu-Habitat. (2016). Índice de las Ciudades Prósperas en la Republica Mexicana; Reporte nacional de tendencias de la prosperidad urbana en México. México:Onu-Habitat.

Ramirez, K. (2009). Espacio público y ciudadanía en la ciudad de México percepciones, apropiaciones y prácticas sociales en Coyoacán y su centro historico. México: Porrua.

Ramírez, J. y Safa, P. (2010). Metropolis, asociaciones vecinales y megaproyectos urbanos; el caso de jardines del sol y la ciudadela en Zapopan. Guadalajara: ITESO.

Diario Oficial de la Federación. (2014). Sistema Urbano Nacional. Recuperado de http://dof.gob.mx/nota_detalle.php?codigo=5342867\&fecha=30/04/2014.

Tristan, V. (2020). Análisis del uso del espacio público desde la habitabilidad y dinámicas urbanas contemporáneas: el caso de colonia Mitras centro en la ciudad de Monterrey. (Tesis de maestría). Facultad de Arquitectura, Universidad Autónoma de Nuevo León, México.

Turner, B. S. (1990). Outline of a Theory of Citizenship. Sociology, 24(2), 189-217. Recuperado de http://www.jstor.org/stable/42853652. 